\title{
UNCERTAINTIES CONCERNING THE FREE VIBRATION OF INHOMOGENEOUS ORTHOTROPIC REINFORCED CONCRETE PLATES
}

\author{
Vahid Lal SHAHSAVAR ${ }^{*}$, Samira TOFIGHI ${ }^{2}$
}

\section{Abstract}

Analyzing nearly collapsed and broken structures gives good insights into possible architectural and engineering design mistakes and faults in the detailing and mismanagement of a construction by building contractors. Harmful vibration effects of construction operations occur frequently. The background reviews have demonstrated that the problem of the vibration serviceability of long-span concrete floors in buildings is complex and interdisciplinary in nature. In public buildings, floor vibration control is required in order to meet Serviceability Limit States that ensure the comfort of the users of a building. In industrial buildings, machines are often placed on floors. Machines generate vibrations of various frequencies, which are transferred to supporting constructions. Precision machines require a stable floor with defined and known dynamic characteristics. In recent years there has been increasing interest in the motion of elastic bodies whose material properties (density, elastic moduli, etc.) are not constant, but vary with their position, perhaps in a random manner. Concrete is a non-homogeneous and anisotropic material. Modeling the mechanical behavior of reinforced concrete $(R C)$ is still one of the most difficult challenges in the field of structural engineering.

One of several methods for determining the dynamic modulus of the elasticity of engineering materials is the vibration fre-

\section{INTRODUCTION}

Most structural failures are the result of an error made by one of the people involved in the great number of steps between the original idea and the completion of the final structure. For reinforced concrete constructions, inadequate plate designs and overweight structures are the main causes of fatal building and bridge failures and the related human victims. Floor vibrations affect not only the comfort of the occupants but also sensitive equipment that might be on the floor, especially in industrial and laboratory settings (Bazant, 2000). Exces-
Address

1 Azerbaijan University of Architecture and Construction, No 34, Zaal St., Roodaki Av., Urmia, IRAN. e-mail: vahidlal@yahoo.com. P. code: 57191-67934

2 Azad University of Mahabad, Mahabad, 59136 Azarbayjan-eGharbi, Iran

Corresponding author: vahidlal@yahoo.com

\section{Key words}

- Free vibration,

- concrete plates,

- orthotropic and inhomogeneous behavior,

- uncertainly analysis.

quency procedure. In this method, the required variables except for the modulus of elasticity are accurately and certainly determined.

In this research, the uncertainly analysis of the free vibration of inhomogeneous orthotropic reinforced concrete plates has been investigated. Due to the numerous outputs obtained, the software package has been written in Matlab, and an analysis of the data and drawing related charts has been done. 
1964; Leissa, 1969). Although rectangular plates of variable thicknesses have received much less attention than uniform plates, they have still been investigated quite intensively.

From 1969 to 1987, Leissa et al. compiled some works performed in the field of homogeneous and non-homogeneous plates. An excellent survey of work up to 1987 on the vibration of homogeneous isotropic and anisotropic plates of various geometries was conducted by Gorman 1982; Timoshenko and Woinowsky, 1984; Tomar et al., 1982, 1983, 1984; and Shames and Dym, 1985. Since 1987, studies of homogeneous rectangular orthotropic plates have been carried out by several researchers. Krauberger et al. analyzed the transverse vibrations of non-homogeneous rectangular plates of uniform thicknesses using boundary characteristic orthogonal polynomials. Poisson's ratio has been assumed to be constant (Krauberger et al, 2012; NSW, 2006).

A designer must evaluate the critical and free vibration frequencies of plates. In reality, some of the design parameters in a structural analysis may be disregarded, which can lead to uncertainties. In this research, an uncertainty analysis of the free vibration of inhomogeneous orthotropic reinforced concrete plates has been investigated. To do this, a sensitivity and uncertainly analysis of free vibrations using various parameters, such as the modulus of elasticity in two directions (the orthotropic coefficient), compression/tension forces, the length to width ratio, density variations of concrete, and inhomogeneous coefficients, has been investigated. Studies have been done on a set of concrete plates with and without inhomogeneous properties. The plate sections are generally square or rectangular (Berczyński, 2011; Elishakoff, 2005).

\section{INHOMOGENEOUS BEHAVIOR OF CONCRETE}

Material properties affect the critical values of the free vibration frequencies of plates. There are some factors which affect the mechanical factors of concrete in one dimension that are not uniform and isotropic in the other one. These factors have an effect on the concrete elasticity modulus, Poisson coefficient and regular relations of the vibration frequencies of plates. The material modeling of reinforced concrete, which generally consists of three phases, i.e., cement mortar, aggregate grains and reinforcing steel bars, is a strong compromise between the structural phenomena and material parameters available. In a structural analysis, reinforced concrete materials are modeled as a macroscopically homogeneous material with response influences on each of the phases (Mostofinejad, 2006).

\subsection{Strength of Concrete}

Concrete strength is counted as one of the most important parameters for material properties in a reinforced concrete structure's design. Using the same mixing, concrete could achieve different compressive strength results in different situations. The compressive strength of concrete depends on some main factors, for example, the aggregate grading, the aggregate/cement ratio as well as the water/cement ratio. These also depend on some minor factors or site factors, for example, grout leakage, poor compaction (the influence of gravity force on the concentration of layers and the type of concrete compression vibrations), segregation, grading limits, poor curing, and chemical attacks such as chlorides, sulfates, carbonation, alkali-silica reactions, and acids. Some studies show that humidity and a good temperature in concreting after 180 days can increase the concrete's strength up to 3 times that of uncured concrete. In those seasons with direct sunshine, the temperature increases, and the humidity of the concrete section decreases by 2 or 3 degrees. This factor is important when considering the surface areas of slabs.

\subsection{Cracks in Concrete Sections}

The most important issues raised regarding serviceability are the cracking and deflection of RC structures. It is obvious that any weakness of the concrete in tension at the time of the utilization of a structure will result in cracks in the RC elements. Although the existence of such cracks does not pose any threat to the structure, if their width is not monitored and controlled, there is a possibility of humidity penetrating the $\mathrm{RC}$ together with harmful ions and, consequently, causing the corrosion of the bars. This will cause a sense of insecurity among the users of a structure.

Concrete exhibits a large number of micro-cracks, especially at the interface between coarser aggregates and mortar, even before the application of any external loads. The presence of these micro-cracks has a great effect on the mechanical behavior of concrete, since their propagation (concrete damage) during loading contributes to the nonlinear behavior at low stress levels and causes volume expansion close to failure. Many of these micro-cracks are initially caused by the segregation, shrinkage or thermal expansion of the mortar. Some micro-cracks may develop during loading because of the difference in stiffness between the aggregates and mortar (Mostofinejad, 2006, Tim Gudmand-Høyer and Lars Zenke Hansen, 2002).

\subsection{Density of Concrete}

In practice, with a change in gradation and concrete compaction, the density and compressive strength of concrete change (Table I). It may happen that with a small change in density and without any external interference, the compressive strength of concrete decreases due to a decrease in density (Shahsavar, 2011).

\subsection{Static Modulus of Elasticity}

A reinforced concrete structure may be subjected to four basic types of actions: bending, axial load, shear and torsion. All of these actions can, for the first time, be analyzed and designed by a single unified theory based on the three fundamental principles of the mechanics of materials.

Material properties can be defined through concrete strength and the modulus of elasticity as proposed in different national building codes through various formulas for the same values of concrete strength. The modulus of the elasticity of concrete is a key factor for estimating the deformation of buildings and members, as well as a fundamental factor in determining the modular ratio $n$, which is used for the design of the sections of members subjected to flexure. The modulus of the elasticity of concrete is frequently expressed in terms of compressive strength. The slope of the stress-strain curve is defined as the elasticity modulus in concrete.

This modulus relates to the kind of concrete, the age of the concrete and the speed in loading, the concrete properties, and mixing percent. More importantly, it relates to the definition of the concrete's elasticity modulus. According to Table I, the two factors, compressive strength and density, are related to the elasticity modulus. In concreting, if one is careful about how to compact the concrete and complete it, the concrete will have great compressive strength. So, all the aspects that influence compressive strength and density have a direct influence on the elasticity modulus too.

The compressive strength of steam-cured concrete is not as high as that of similar concrete continuously cured under moist conditions at moderate temperatures. Also, the modulus of elasticity $E_{c}$ of steamcured specimens may vary from that of specimens moist-cured at nor- 
Tab. 1 Material properties as a function of the compressive strength.

\begin{tabular}{|c|c|c|c|}
\hline No. & Code & Formula & References \\
\hline 1 & ACI-2008 & $\mathrm{Ec}=4.73 \sqrt{\mathrm{f}_{\mathrm{c}}^{\prime}}$ & American Concrete Institute \\
\hline 2 & CEB-90 & $E c=10\left(f_{c}^{\prime}+8\right)^{1 / 3}$ & $\begin{array}{c}\text { Euro-International Concrete } \\
\text { Committee }\end{array}$ \\
\hline 3 & TS-500 & $\mathrm{Ec}=3.25 \sqrt{\mathrm{f}_{\mathrm{c}}^{\prime}}+14$ & Turkish Standard Committee \\
\hline 4 & IDC-3274 & $\mathrm{Ec}=5.7 \sqrt{\mathrm{f}_{\mathrm{c}}^{\prime}}$ & Italian Design Council \\
\hline 5 & GBJ-11-89 & $\mathrm{Ec}=\frac{10^{2}}{\left[2.2+\frac{34.7}{\mathrm{f}_{\mathrm{c}}^{\prime}}\right]}$ & Chinese Design Council \\
\hline 6 & ABA & $\mathrm{Ec}=5.0 \sqrt{\mathrm{f}_{\mathrm{c}}^{\prime}}$ & Iranian Concrete Code \\
\hline 7 & Mos-2005 & $\mathrm{Ec}=8.3\left(\mathrm{f}_{\mathrm{c}}^{\prime}\right)^{0.35}$ & $\begin{array}{l}\text { Prof. Mostofinejad, Davood } \\
\text { (Ellingwood-Tallin, 1984) }\end{array}$ \\
\hline
\end{tabular}

mal temperatures. The modulus of elasticity for concrete is sensitive to the modulus of elasticity of the aggregate and may differ from the specified value. The measured values typically range from 80 to 120 percent of the specified value.

It was concluded that the effect of the variations of $E_{c}$ is twofold: direct effect and indirect effects. The direct effect was discussed earlier as $E_{c}$ being part of the stiffness matrix of the structure and therefore significantly affecting the natural period and displacement of the structure under dynamic and static loading. The indirect effect shows up in calculating the cracked section's moment of inertia of the structure; using a higher modulus of elasticity in the elastic analysis will result in a stiffer structure along with a lower displacement demand and, therefore, less cracking of the concrete. A reduction of the cracking of concrete will result in an increase in the effective (cracked) moment of inertia (Shahsavar, 2011; Mostofinejad, 2006).

There are various research works available in the literature for determining the sensitivity of the modulus of elasticity to the vibrations of plates. In this study, a procedure was used to investigate the effect of material uncertainties. In the present study, seven different design codes were selected and considered in the analyses (Table II). The relationships of $f_{c}^{\prime}$ and $E_{c}$ are expressed in MPa and in GPa, respectively. The relationship curves, the modulus of elasticity, and various concrete strengths for different design codes are shown in Fig. 1.

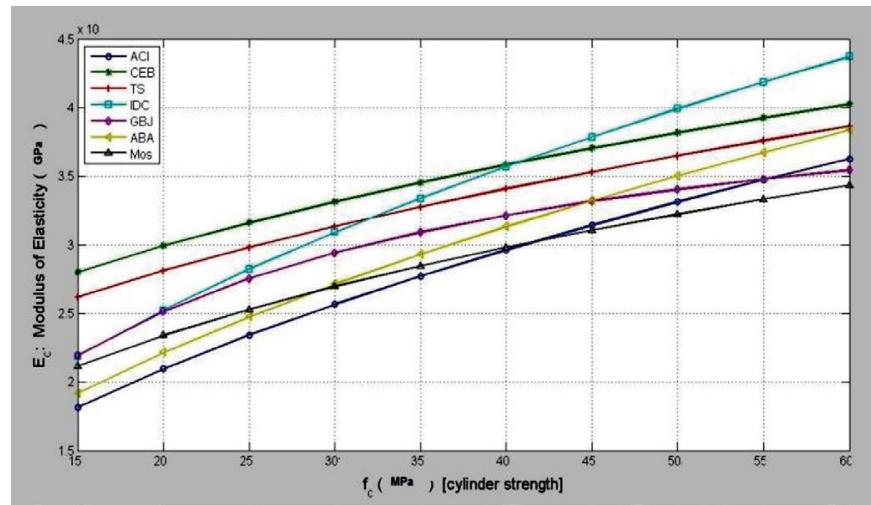

Fig. 1 Changes in the modulus of elasticity in various concrete strengths for different design codes.
Tab. 2 Elastic modulus values for a given concrete strength.

\begin{tabular}{|c|c|c|c|c|}
\hline \multirow[b]{3}{*}{ References } & \multicolumn{4}{|c|}{ Ec, modulus of elasticity (Gpa) } \\
\hline & $f^{\prime} c=25$ & $f^{\prime} c=25$ & $f^{\prime} c=35$ & $f^{\prime} c=35$ \\
\hline & 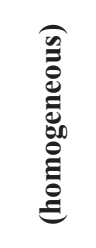 & 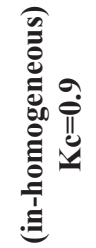 & 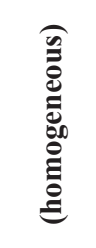 & 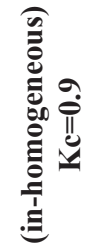 \\
\hline CI-2008 & 23,4 & 22,2 & 27,7 & 26,3 \\
\hline CEB-90 & 31,6 & 30,8 & 34,5 & 35,8 \\
\hline TS-500 & 29,8 & 29 & 32,8 & 31,8 \\
\hline IDC-3274 & 28,2 & 26,8 & 33,4 & 31,7 \\
\hline GBJ-11-89 & 27,5 & 26,4 & 30,9 & 29,9 \\
\hline ABA & 24,8 & 23,5 & 29,3 & 27,8 \\
\hline Mos-2005 & 25,3 & 24,4 & 28,4 & 27,4 \\
\hline
\end{tabular}

\subsection{Dynamic Modulus of Elasticity}

The dynamic modulus is consistently higher than the static modulus. Static and dynamic moduli follow different mixture behaviors in composite materials such as concrete. It is this difference that may cause the dynamic moduli to be higher than the static moduli in concrete. Non-destructive dynamic methods can be used to estimate in-place $E$, but the meaning of the dynamic modulus obtained is uncertain because $E_{d}$ is known to be different (higher) from that obtained by the direct static testing of a cylinder drawn from the structure. Concrete is expected to show a nonlinear dependence between stress and strain, even at low values of deformation caused by quasi-static tests and dynamic tests based on stress-wave propagation (ACI-CRC Final Report, 2008).

Several attempts have been made to correlate static $(E)$ and dynamic $\left(E_{d}\right)$ moduli for concrete $[1,4]$. The simplest of these empirical relations was proposed by Lydon and Balendran (Neville, 1997):

$$
E=0.83 E_{d}
$$

Another empirical relationship for concrete's elastic moduli was proposed by Swamy and Bandyopadhyay and is now accepted as part of the British testing standard BS8110, Part 2:

$$
E=1.25 E_{d}-19
$$

where both units of $E$ and $E_{d}$ are in GPa.

For both lightweight and normal concretes, Popovics suggested a more general relationship between the static and dynamic moduli as a function of the density of the concrete:

$$
E_{s}=k E_{d}^{1.4} \rho^{-1}
$$

where $k=0.23$ for units of psi and $\rho$ is density, lbs/ft3 (Popovics, 1975).

\subsection{Flexural Stiffness (EI and D)}

In defining the concrete's parameters analytically, the main problem is the choice of stiffness $E I$, which reasonably approximates the 
variations in stiffness due to cracking, creep, and the nonlinearity of the concrete stress-strain curve.

It is obvious that due to the non-homogeneous quality of concrete, its tension resistance is not absolutely consistent in any given length. The simple equations for the strength of material can be used to calculate the instantaneous deflection in a steel flexural element. However, when calculating the deformation of one beam of $\mathrm{RC}$ or a concrete plate, one encounters the problem of determining the stiffness of its section. In fact, in a beam/plate with a steel section, the elasticity module is stable during loading and reaching the yielding limit. Furthermore, under bending, the steel section will present its ultimate strength both in compression and tension; therefore, its moment of inertia and, consequently, its bending stiffness will be fixed. However, in a beam/ plate with a concrete section, both the elasticity module and the moment of inertia will vary during loading due to cracking. In such a case, it is important that the change in the moment of inertia be proportional to the change in the amount of load along the beam/plate.

As previously discussed, calculating the instantaneous deflection faces two major problems: stiffness change in the section of the beam/plate at the time of loading because of tension stiffening, and stiffness change in the length and other various areas of the beam/ plate, not to mention that the elasticity modulus of concrete varies depending on the amount of load. To simplify the calculation of deformations in RC members, the concept of effective flexural stiffness $(E I)_{e f f}$ or $D$ is used. If the elasticity modulus of concrete is considered constant (its amount equaling the slope which connects the point of origin to the analogous tension point $0.4 f c^{\prime}$ in the stress-strain), and if the moment-deformation curve of the member is assumed to be a two-line curve, the effective moment of inertia can be considered uniform along the member. This effective moment of inertia will be a function of the moment of inertia of the uncracked section $\left(I_{u c}\right)$ and the moment of inertia of the uncracked section $\left(I_{c r}\right)$, Fig. 2.

According to his broad experimental studies, Branson has proposed the following equation to determine the effective moment of the inertia of a beam when calculating deflections.

$$
I_{e}=\left(\frac{M_{c r}}{M_{a}}\right)^{a} I_{u c}+\left[1-\left(\frac{M_{c r}}{M_{a}}\right)^{a}\right] I_{c r}
$$

In the above equation, $I_{u c}$ and $I_{c r}$ are the moment of inertia of the uncracked section and the moment of inertia of the cracked section, respectively; $M_{c r}$ and $M_{a}$ represent the cracking moment of a section and the maximum flexural moment of the section. Also, a is a constant coefficient, which is $\mathrm{a}=4$ for sections under a consistent moment and is used to include the tension stiffening of concrete in the equation; furthermore, $\mathrm{a}=3$ is proposed by Branson for simply supported beams and is used to include the tension stiffening of concrete and also the variations in the stiffness along beams (Berczyński, 2011; Mostofinejad,2006).

\section{SERVICABILITY LIMIT STATES AND VIBRATIONS OF SLABS}

Serviceability limit states in structural engineering occur when the function of the structure is disrupted because of local minor damage, deterioration of structural or nonstructural components, or excessive structural movement. Excessive structural deflections are a main source of unserviceability in buildings. Static deflections may affect the appearance or efficiency of structural and nonstructural elements and mechanical equipment. Dynamic deflections may lead to occupant discomfort and the impaired function of sensitive mechanical equipment, especially if resonance occurs (Lal et al., 2010; Sharma et al., 2012).

A floor is an integral part of practically every modern industrial, commercial or residential building. As expectations of building users who are in everyday contact with the floors rise, so the performance of floor structures in day-to-day service is becoming increasingly important. Most of the knowledge about the vibration performance of suspended floors in buildings has been gained during this century. Modern large span floors and lightweight floors show a tendency to vibrate under service conditions.

Floor structures are designed for ultimate limit states and the following serviceability limit state criteria:

- Ultimate limit states are those related to strength and stability;

- Serviceability limit states are mainly related to vibrations and hence are governed by stiffness, masses, damping and excitation mechanisms.

When considering concrete floors as vibration paths, caution is required, as closely spaced modes of vibration tend to occur in orthotropic building floors having a repetitive geometry. Typical receivers of floor vibrations are its human occupants or vibration-sensitive equipment. The characterization of humans as floor vibration receivers is probably the most difficult aspect of the floor vibration serviceability problem.

Special attention should be paid in each case to the dynamic properties of a designed construction. In public buildings, floor vibration control is required due to meet Serviceability Limit States that ensure the comfort of the users of a building. A review of vibration sources found that humans are the most relevant cause of excitation of building floors, which is practically impossible to isolate effectively. Moreover, mathematical models simulating human-induced excitation, such as 'near periodic' walking, running or jumping, do exist, but caution is required when using them on lightly damped floor structures. In this case, an assumption of pure resonance may lead to a significant overestimation of responses due to the inability of humans to generate perfectly periodic forces.

Where occupants can detect vibrations in buildings, this may potentially impact on their quality of life or working efficiency. In con-
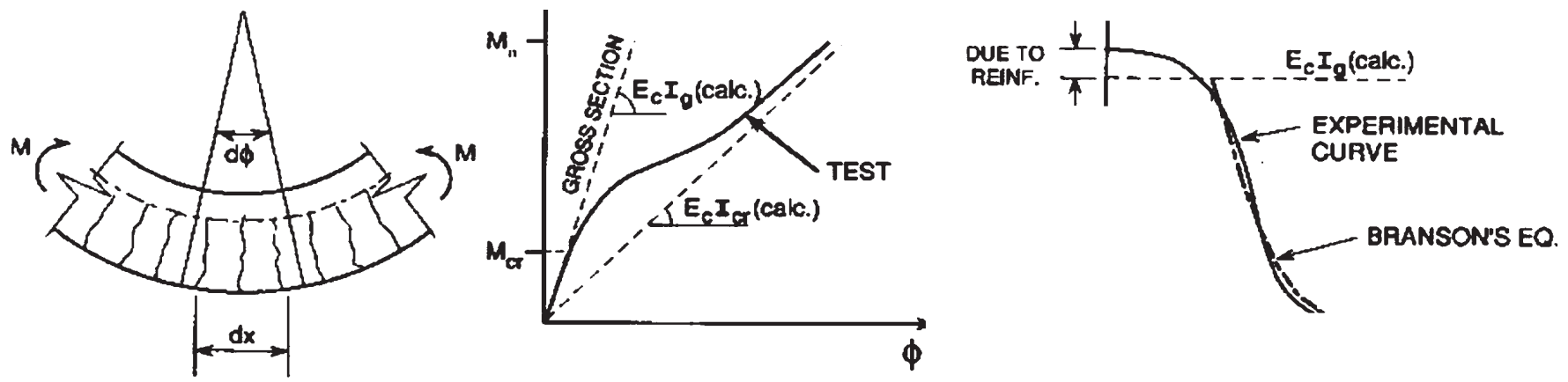

Fig. 2 Variation of the effective stiffness of the cracked and uncracked sections. 
trast, people tolerate much higher vibration values in vehicles than in buildings. Individuals can detect building vibration values that are well below those that can cause any risk of damage to the building or its contents. The level of vibration that affects an amenity is lower than that associated with building damage.

Vibration serviceability problems were first observed to occur to a greater extent in steel joist-concrete composite floors in the 1950s and 1960s. In 1988, when vibration serviceability due to the above-mentioned design trends was becoming an issue for a much wider range of floors, including concrete floors, the following three general research tasks requiring further work were identified (Galambos, 1988):

1. to quantify a rationally human response to vibrating floors as expressed by the emotions of annoyance and fear,

2. to define rationally the excitation input from human activity on a floor which causes vibrations, and

3. to effectively define the structural dynamic model of a real floor in service, including the determination of more reliable damping.

The American Society of Civil Engineers (ASCE) Standard 7-95 (ASCE, 1995) defines the serviceability limit states (SLS) as: "...conditions in which the functions of a building or other structure are impaired because of local damage, deterioration or deformation of building components or because of occupant discomfort." Occupant discomfort is mainly caused by the vibration motions of a building as a whole or of individual building floors. Whereas wind typically excites horizontal vibrations of (tall) buildings, the occupant activities are the main source of vertical floor vibrations (Ellingwood, 1996).

In bridge engineering, dynamic load is common, and particular attention should be paid to small and medium-span beam bridges that are found along high-speed rail tracks where trains can travel with speeds exceeding $300 \mathrm{~km} / \mathrm{h}$.

For the prediction of floor vibrations several dynamic floor characteristics need to be determined. Every structure has its specific dynamic behavior with regard to the vibration mode's shape and duration $\mathrm{T}[\mathrm{s}]$ of a single oscillation. Each structure has as many natural frequencies and associated mode shapes as degrees of freedom. They are commonly sorted by the amount of energy that is activated by the oscillation. Therefore the first natural frequency is that on the lowest energy level and is thus the most likely to be activated (Ellingwood-Tallin, 1984; Mali-Singru, 2012; Krauberger et al., 2012; Lal et al., 210; Shahsavar, 2011; Luo et al., 2012; Svinkin, 2004; Mostofinejad, 2006; Pavic-Reynolds, 2007; Sharma et al, 2012; Hosseini-Hashemi et al., 2010).

\subsection{Limited Area of Vibration}

A floor is a sophisticated, dynamic system. As a continuous structure it has an infinite number of modes of vibration (degrees of freedom), but only the first few modes contain almost all the vibration energy of the floor. The first mode (fundamental mode) has the lowest natural frequency, the largest movement, and possesses the lion's share (up to $80 \%$ in some applications) of the vibration energy. Frequently, quieting this mode alone lowers the overall floor vibrations to an acceptable level.

Human activities excite floors at the first few natural frequencies. Such activities usually have forcing frequencies in a range of 1.0 to $3.0 \mathrm{~Hz}$. For instance, walking at a pace of about $2 \mathrm{~Hz}$ perturbs a flexible floor and its higher order harmonics at that frequency. When a harmonic of occupants' activities is very close to or matches one of the natural frequencies of the floor, it makes the floor resonate at that frequency, causing excessive vibrations. As an example, in an office building with reported walking-induced vibrations, the average walk- ing pace of the occupants was measured at around $2.35 \mathrm{~Hz}$. The first resonant frequency of the floor was measured at about $4.7 \mathrm{~Hz}$. Having the 2nd harmonic of walking exciting the first resonant frequency of the floor caused excessive vibration.

People are known to be very sensitive to floor vibrations, e.g., vibrations with an amplitude as small as 0.004 inch $(0.1 \mathrm{~mm})$ can cause aggravation. Floors that are most disturbing to the occupants often have low resonant frequencies; residential and office building floors having their fundamental frequency usually in the range of 3.5 to 8 $\mathrm{Hz}$ fall in this category. This might be because the natural frequencies of the internal human organs are also in the same frequency range, i.e., 4 to $8 \mathrm{~Hz}$. That is, floor resonance can cause the internal organs of the occupants to resonate, resulting in an uneasy and irritating feeling (NSW, 2006; Pavic-Reynolds, 2007). In Table III, some examples of types of vibration have been demonstrated.

Tab. 3 Examples of types of vibration.

\begin{tabular}{|c|c|c|}
\hline $\begin{array}{c}\text { Continuous } \\
\text { vibration }\end{array}$ & $\begin{array}{c}\text { Impulsive } \\
\text { vibration }\end{array}$ & Intermittent vibration \\
\hline $\begin{array}{c}\text { Machinery, } \\
\text { steady road } \\
\text { traffic, } \\
\text { continuous } \\
\text { construction } \\
\text { activity } \\
\text { (such as } \\
\text { tunnel boring } \\
\text { machinitient: } \\
\text { create up to } 3 \\
\text { distinct vibration } \\
\text { events in an } \\
\text { assessment period, } \\
\text { e.g., occasional } \\
\text { dropping of } \\
\text { heavy equipment, } \\
\text { occasional loading } \\
\text { and unloading. }\end{array}$ & $\begin{array}{c}\text { Trains, nearby intermittent } \\
\text { construction activity, heavy } \\
\text { vehicles passing, forging } \\
\text { machines, impact pile driving, } \\
\text { jack hammers. Where the } \\
\text { number of vibration events in } \\
\text { an assessment period is three or } \\
\text { fewer, this would be assessed } \\
\text { against impulsive vibration } \\
\text { criteria. }\end{array}$ \\
\hline
\end{tabular}

Resonance has been ignored in the design of floors and footbridges until recently. Approximately 30 years ago, problems arose with vibrations induced by walking on steel-joist supported floors that satisfied traditional stiffness criteria. Since that time much has been learned about the loading function due to walking and the potential for resonance. More recently, rhythmic activities, such as aerobics and high-impact dancing, have caused serious floor vibration problems due to their resonance (Luo et al., 2012; Yin, 2013).

\section{STUDY OF THE UNCERTAINTIES OF THE VIBRATION OF CONCRETE PLATES}

A rectangular plate of a certain material is assumed, as seen in Fig. 3. Forces $P_{1}$ and $P_{2}$ are applied to it in two main directions, and the lateral load $P_{(x)}$ can affect it dynamically. The plate is simply supported at its 4 edges, and the general equation of its bending movement is presented in equation, in which $L(w)$ is a function defined in equation, and $W$ is the equation of the plate displacement compared to the $\mathrm{X}$ and $\mathrm{Y}$ axes. $P_{1}$ and $P_{2}$ are the compressive and/or tensile forces in directions 1 and 2, respectively, which are applied to the plate at its two ends (Fig. 3). $H_{0}$ is the uncracked thickness of the plate, and $\mathrm{h}$ is its effective thickness, which is determined through the cracking criteria defined for the RC elements. $P_{(x)}$ is the likely transverse force applied to the plate and varies by time, which is shown as t. $\omega$ is the angular frequency, which is the desired value to be obtained from equation . 


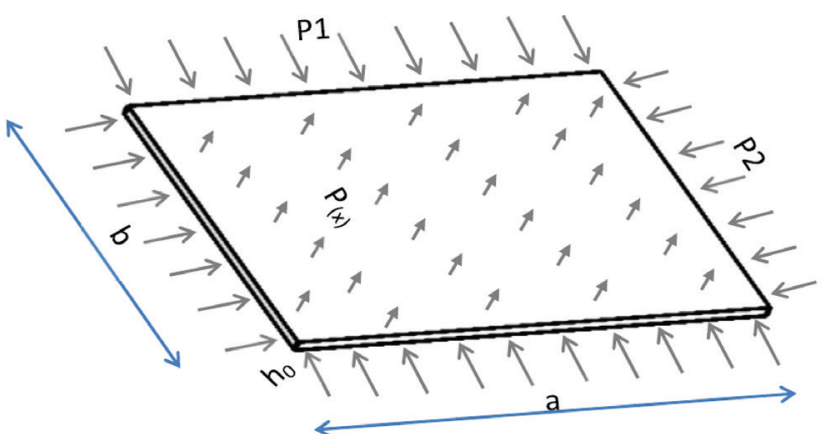

Fig. 3 Assumed rectangular plate with loads applied.

$$
\begin{gathered}
L(W)+p_{1} \frac{\partial^{2} W}{\partial x^{2}}+p_{2} \frac{\partial^{2} W}{\partial y^{2}}+h \rho_{0} \varphi(x) \frac{\partial^{2} W}{\partial t^{2}}=p(x) e^{i \alpha} \\
L(W)=f(x)\left[D_{1}^{0} \frac{\partial^{4} W}{\partial x^{4}}+\left(D_{k}^{0}+v_{2} \cdot D_{1}^{0}+v_{1} \cdot D_{2}^{0}\right) \frac{\partial^{4} W}{\partial x^{2} \partial y^{2}}+\right. \\
\left.+D_{2}^{0} \frac{\partial^{4} W}{\partial y^{4}}\right]+D_{1}^{0}\left[\frac{\partial^{2} f}{\partial x^{2}}\left(\frac{\partial^{2} W}{\partial x^{2}}+v_{2} \frac{\partial^{2} W}{\partial y^{2}}\right)+\right. \\
\left.+2 \frac{\partial f}{\partial x}\left(\frac{\partial^{3} W}{\partial x^{3}}+v_{2} \frac{\partial^{3} W}{\partial x \partial y^{2}}\right)\right]+D_{k}^{0}\left[\frac{\partial f}{\partial x} \cdot \frac{\partial^{3} W}{\partial x^{2} \partial y}\right] \\
E_{1}=E_{1}^{0} f(x), \mathrm{E}_{2}=E_{2}^{0} f(x), G=G_{0} f(x) ; \rho=\rho_{0} \psi(x) \\
D_{1}^{0}=\frac{E_{1}^{0} h^{3}}{12\left(1-v_{1} v_{2}\right)} ; \mathrm{D}_{2}^{0}=\frac{E_{2}^{0} h^{3}}{12\left(1-v_{1} v_{2}\right)} ; \mathrm{D}_{k}^{0}=\frac{G_{0} h^{3}}{12}
\end{gathered}
$$

Where $D^{0}{ }_{1}$ and $D^{0}{ }_{2}$ are the flexural stiffness of the plate around directions 1 and 2, respectively, and $D^{0}{ }_{k}$ represents its total stiffness. $v_{1}$ and $v_{2}$ are the Poisson's coefficient of the material in two main directions. In order to solve the vibration equation of the plate, we assume:

$$
W(x, y)=V(x, y) e^{i \omega x}
$$

Through the placement of equation in equation (5), equation is obtained:

$$
L(V)+\mathrm{p}_{1} \frac{\partial^{2} V}{\partial x^{2}}+\mathrm{p}_{2} \frac{\partial^{2} V}{\partial y^{2}}-\omega^{2} \rho_{0} \psi(x) V-p(x)
$$

Where $L(v)$ equals:

$$
\begin{aligned}
L(V) & =f(x)\left[D_{1}^{0} \frac{\partial^{4} V}{\partial x^{4}}+\left(D_{k}^{0}+v_{2} \cdot D_{1}^{0}+v_{1} \cdot D_{2}^{0}\right) \frac{\partial^{4} V}{\partial x^{2} \partial y^{2}}\right. \\
& \left.+D_{2}^{0} \frac{\partial^{4} V}{\partial y^{4}}\right]+D_{1}^{0}\left[\frac{\partial^{2} f}{\partial x^{2}}\left(\frac{\partial^{2} V}{\partial x^{2}}+v_{2} \frac{\partial^{2} V}{\partial y^{2}}\right)+\right. \\
+ & \left.2 \frac{\partial f}{\partial x}\left(\frac{\partial^{3} V}{\partial x^{3}}+v_{2} \frac{\partial^{3} V}{\partial x \partial y^{2}}\right)\right]+D_{k}^{0}\left[\frac{\partial f}{\partial x} \cdot \frac{\partial^{3} V}{\partial x^{2} \partial y}\right]
\end{aligned}
$$

When the plate has simply supported connections at its 4 edges, the boundary conditions for solving the above equation are the following:

$$
V=0, \quad \frac{\partial^{2} V}{\partial x^{2}}=0 \quad\{x=0 \quad x=a\}
$$

$$
V=0, \quad \frac{\partial^{2} V}{\partial y^{2}}=0 \quad\{y=0 \quad y=b\}
$$

As obviously seen, it is not easy to accurately solve equation , which depends on the two variable functions $f_{(x)}$ and $w_{(x)}$. Where $f_{(x)}$ is the variable function dependent on the surface coordinates of the plate and shows the inhomogeneous mechanical characteristics of the plate body, $w_{(x)}$ is the equation of the plate displacement compared to the $X$ and $Y$ axes. To achieve a solution of the equation with fewer errors, approximate solution methods such as the proven and effective Bubnov-Galerkin method will be used. According to the above method:

$$
V(x, y)=V_{0} \varphi_{1}(x) \varphi_{2}(x)
$$

In this equation, $\varphi_{1(x)}$ and $\varphi_{2(y)}$ are adapted to the boundary conditions in equation. In the next, for determining the free frequency, the forced vibration will be ignored, and $P_{(x)}$ will be considered as zero. Therefore, we have equation, and following this, equation :

$$
\begin{gathered}
\int_{0}^{a} \int_{0}^{b}\left[\left(\varphi_{1}, \varphi_{2}\right)+p_{1} \frac{\partial^{2} \varphi_{1}}{\partial x^{2}} \varphi_{2}(y)+p_{2} \frac{\partial^{2} \varphi_{2}}{\partial y^{2}} \varphi_{1}(x)-\right. \\
\left.-\omega^{2} \rho_{0} h \psi(x) \varphi_{1}(x) \varphi_{2}(y)\right] \varphi_{1}(x) \varphi_{2}(y) \mathrm{dxdy}=0 \\
\omega^{2}=\frac{\int_{0}^{a} \int_{0}^{b}\left[\left(\varphi_{1}, \varphi_{2}\right)+p_{1} \frac{\partial^{2} \varphi_{1}}{\partial x^{2}} \varphi_{2}(y)+p_{2} \frac{\partial^{2} \varphi_{2}}{\partial y^{2}} \varphi_{1}(x)\right] \varphi_{1}(x) \varphi_{2}(y) \mathrm{dxdy}}{\rho_{0} h \int_{0}^{a} \int_{0}^{b} \psi(x) \varphi_{1}^{2}(x) \varphi_{2}^{2}(y) \mathrm{dxdy}}
\end{gathered}
$$

As mentioned before, $f_{(x)}$ and $v_{(x)}$ are variable functions dependent on the surface coordinates of the plate and show the inhomogeneous mechanical characteristics of the plate body. To solve the eigenvalues and determine the angular frequency, we will have to assume an equation for them:

$$
\begin{aligned}
& f(x)=1+\varepsilon \frac{x}{a} ; \quad \psi(x)=1+\mu \frac{x}{a} ; \quad \varepsilon \in[0,1] ; \quad \mu \in[0,1] \\
& \varphi_{1}(x)=\sin \alpha_{m} x ; \quad \varphi_{2}(y)=\sin \beta_{n} y \quad\left(\alpha_{m}=\frac{m \pi}{a} ; \quad \beta_{n}=\frac{n \pi}{b}\right)
\end{aligned}
$$

Through the placement of equation in equation, we have equation :

$L\left(\varphi_{1}, \varphi_{2}\right)=\bar{D}(1+\varepsilon \bar{x}) \sin \alpha_{m} x \sin \beta_{n} y-D_{1}^{0} \varepsilon\left(\alpha_{m}^{3}+v_{2} \beta_{n}^{2} \alpha_{m}\right) \alpha$

$\left(\cos \alpha_{m} x \cdot \sin \beta_{n} y\right)-2 D_{2}^{0} \varepsilon\left(\beta_{m}^{3}+v_{1} \alpha_{m}^{2} \beta_{m}\right) b^{-1} x\left(\sin \alpha_{m} x \cdot \cos \beta_{\text {, }}\right.$

$+D_{k}^{0} \varepsilon\left(\alpha^{-1}\right) \sin \alpha_{n} x \cdot \cos \beta_{m} y \alpha_{n}^{2} \beta_{m}$

$$
\bar{D}=D_{1}^{0} \alpha_{m}^{4}+\left(D_{k}^{0}+v_{2} D_{1}^{0}+v_{1} D_{2}^{0}\right) \alpha_{m}^{2} \beta_{n}^{2}+D_{2}^{0} \beta_{n}^{4}
$$

where $m=n=1$, it will activate the main frequency mode and provide the main vibration frequency of the structure. In this paper, frequency sensitivity and frequency change will be investigated in both states where $m=n=2$ and $m=n=3$.

$$
\omega^{2}=\frac{\bar{D}(1+0,25 \varepsilon)-\alpha_{m}^{2} p_{1}-\beta_{n}^{2} p_{2}}{\rho_{0} h(1+0,25 \mu)}
$$

The above equation shows the angular frequency of the vibration in a rectangular plate which can be inhomogeneous both in terms of density and mechanical characteristics $(E$ and $G)$. At the same time, this plate can also possess the orthotropic property $E_{1} \neq E_{2}$. The inho- 
mogeneous property affecting the density of the plate body is defined with the variable $\mu$. If $\mu=0$, it means that the plate body is homogeneous in terms of density variations. Also, the inhomogeneous property affecting the elasticity modulus or shear modulus is defined with the variable $\varepsilon$. If $\varepsilon=0$, the plate body will be considered homogeneous.

In addition to defining the characteristics of inhomogeneous density, mechanical characteristics, and a plate's orthotropic property, equation 18 also provides the angular frequency of a square plate un$\operatorname{der} P_{1}$ and $P_{2}$ forces.

To determine the sensitivity of the mentioned variables (inhomogeneity of density, inhomogeneity of mechanical characteristics such as $E$ and $G$, orthotropic quality, changes in plate dimensions, changes in the ratio of length to width $b / a$, and changes in applied forces in two main directions $P_{2} / P_{1}$ ), an analysis of their sensitivity will be conducted, and the results will be presented. At every stage, the results will be compared to the angular frequency $\omega_{0}$, which is the angular frequency of the homogeneous isotropic plate without the loads $P_{1}$ and $P_{2}$ being applied.

Regarding the direct relation between angular frequency and variable $D$, equation , it is deemed necessary to investigate the uncertainty and sensitivity of other variables to $D$. If the plate is orthotropic, and if the ratio of the elasticity modulus in the main direction $E_{2} / E_{l}$ varies between 1 and 2, the ratio of $D_{e 2} / D_{e l}$ will vary between 1 and 1.5. Fig. 4 depicts the variation curve of this ratio for the different values of $E_{2} / E_{1}$ and also on various surfaces of the ratio of the length to width $b / a$.

\subsection{Investigating the sensitivity of the angular frequency to variations in the ratio of the loads applied $P_{2} / P_{1}$}

It is assumed that the body of a plate is isotropic and homogeneous and that the angular frequency is indicated with $\omega_{h i}$. The first index $(h)$ and the second index $(i)$ indicate homogeneity and isotropic quality, respectively. In this condition, the ratio of the angular frequency to the base angular frequency $\omega_{0}$ equals

$$
\frac{\omega_{h i}^{2}}{\omega_{0}^{2}}=1-\frac{\alpha_{m}^{2} P_{1}+\beta_{n}^{2} P_{2}}{D}
$$

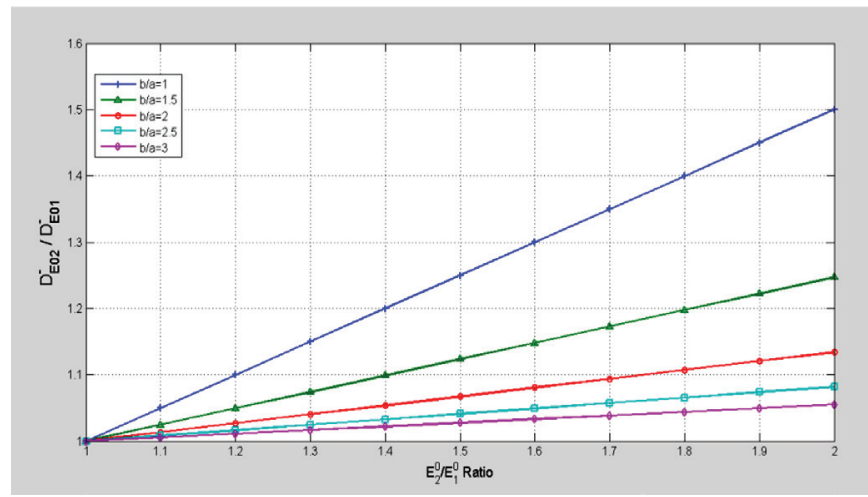

Fig. 4 Variation of $D_{e 2} / D_{e 1}$ ratio to $E_{2} / E_{1}$ ratio changes, various b/a.

In the following, the sensitivity of the powered frequency ratio in the applied loads of $P_{2} / P_{1}$ will be analyzed. Equation has a linear relationship with $P_{2} / P_{1}$; therefore, the powered ratio of the angular frequency will vary in a linear fashion. Fig. 5 depicts the curve for the variations of the powered angular frequency to different ratios of $b / a=1$ and $b / a=3$. As seen in the figure, for the ratios $b / a \geq 2$, the ratio of the powered angular frequency by the ratio of load $P_{2} /$ $P_{1}$, equals 0.885 as a constant amount and does not change with the increase in the ratio of the length to the width, $b / a$.

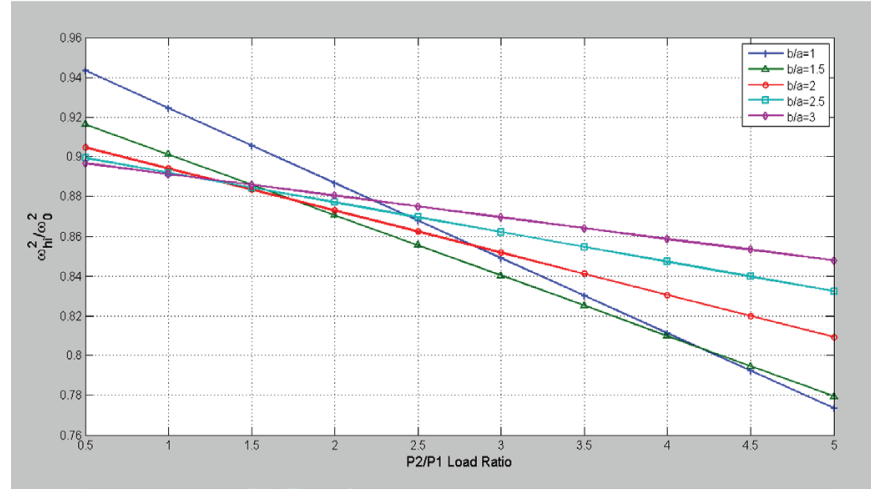

Fig. 5 Variation of $\omega_{h i}{ }^{2} / \omega_{O}{ }^{2}$ ratio to $P_{2} / P_{1}$ ratio changes, various b/a.

\subsection{Investigating the sensitivity of the angular frequency to the density inhomogeneity variable}

For the sensitivity of an angular frequency to the density inhomogeneity variable, it is assumed that the body of the plate is inhomogeneous in terms of density but homogeneous and isotropic in terms of the materials and mechanical characteristics, i.e., the variable $\varepsilon$ is assumed to be 0 , and the variable $\mu$ is investigated based on this assumption. In this case, the angular frequency is represented by $\omega_{i i d^{*}}$ The first index $(i)$ indicates the isotropic quality, and the second one represents the inhomogeneity of the density.

$$
\frac{\omega_{\text {iid }}^{2}}{\omega_{0}^{2}}=\frac{D-\left(\alpha_{m}^{2} P_{1}+\beta_{n}^{2} P_{2}\right.}{D(1+0.25 \mu)}
$$

The following will analyze the sensitivity of the powered angular frequency ratio to the changes in the variable $\mu$ (density inhomogeneity). Equation has a linear relationship with the variable $\mu$; therefore, the powered ratio of the angular frequency will vary in a linear fashion. Fig. 6 depicts the curve for the variations of the powered angular frequency to the density inhomogeneity parameter $(\mu)$. The frequency ratio will decrease, at a certain level of the load ratio $P_{2} / P_{1}$ and with an increase in $\mu$, for a rectangular plate which is inhomogeneous only in terms of density but is isotropic and homogeneous in terms of mechanical characteristics. This indicates that despite the inhomogeneity of the density (with an increasing rate), the plate structure will be flexible; therefore, it will have a higher vibration period. Fig. 6 depicts this softening in a square structure $(b$ $/ a=1)$ for different levels of the load ratio $\left(P_{2} / P_{1}\right)$ and various values of the density inhomogeneity factor $(\mu)$. The figure indicates that with an increase in either of the variables $\mu$ and $P_{2} / P_{1}$, the frequency ratio decreases; therefore, the structure's flexibility increases. Fig. 7 depicts the curves for the variations of the powered angular frequency ratio when the plate has a rectangular shape $(b / a=2)$. Comparing the previous two figures, one can observe that if the plate shape changes to a rectangle of $b / a=2$ from a square, the frequency ratio will decrease as much as 4 percent. Therefore, it can be concluded that in changing other dimensions of the plate, the frequency ratio will not be affected significantly. 


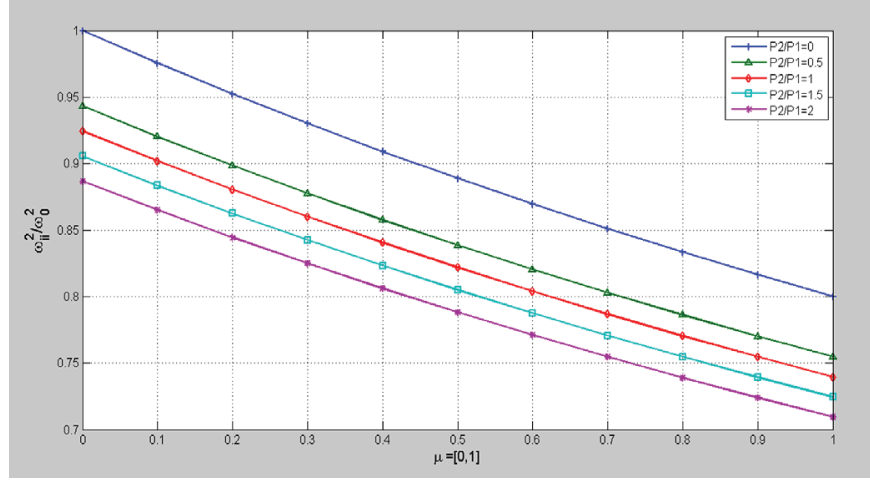

Fig. 6 Variation of $\omega_{i i}{ }^{2} / \omega_{O}{ }^{2}$ ratio to changes of $\mu=[0,1]$, various $P_{2} / P_{1}, b / a=1$.

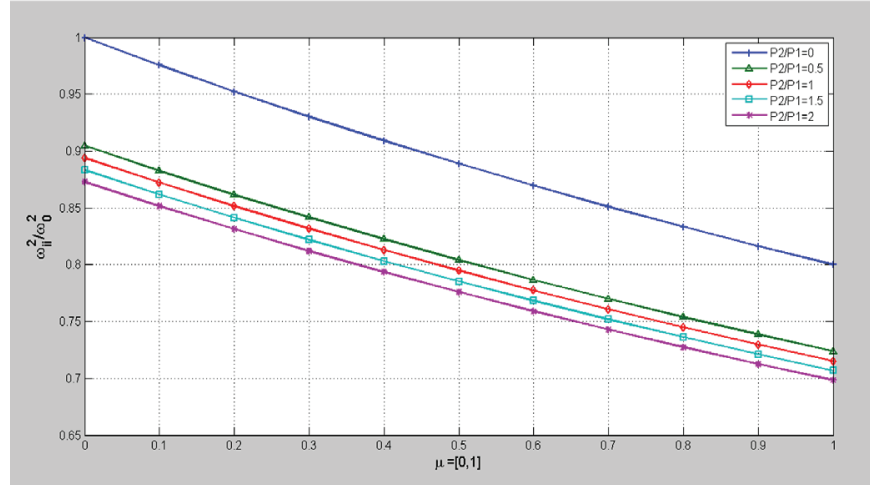

Fig. 7 Variation of $\omega_{i i}{ }^{2} / \omega_{O}{ }^{2}$ ratio to changes of $\mu=[0,1]$, various $P_{2} / P_{1}, b / a=2$.

\subsection{Investigating the sensitivity of the angular frequency to the inhomogeneity variable of the mechanical characteristics of the materials}

\section{A. The plate body is isotropic}

It is assumed that the body of the plate is homogeneous in terms of density but inhomogeneous and isotropic in terms of the materials and mechanical characteristics, i.e., the variable $\mu$ is assumed to be 0 , and the variable $\varepsilon$ is investigated based on this assumption. In this case, the angular frequency is represented by $\omega_{i i}$. The first index (i) indicates the isotropic quality, and the second one represents the inhomogeneity of the mechanical characteristics.

$$
\frac{\omega_{i i}^{2}}{\omega_{0}^{2}}=(1+0.25 \varepsilon)-\frac{\alpha_{m}^{2} P_{1}+\beta_{n}^{2} P_{2}}{D}
$$

The following will analyze the sensitivity of the powered angular frequency ratio on the changes in the variable $\varepsilon$ (the inhomogeneity of the mechanical characteristics of the materials). Equation has a nonlinear relationship with the variable $\varepsilon$; therefore, the powered ratio of the angular frequency will vary in a nonlinear fashion. Fig. 8 depicts the curve for the variations of the powered angular frequency of the inhomogeneity on the mechanical characteristics' parameters. For a square plate the frequency ratio will increase 25 percent with an increase of 100 percent in $\varepsilon$. Fig. 8 depicts this softening in a square plate $(b / a=1)$ for the values of $E_{2} / E_{1}$ equals to 1 .

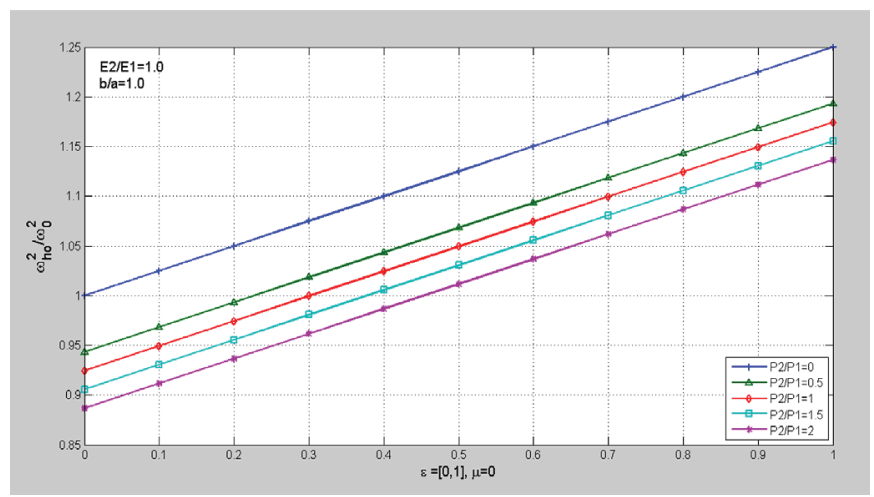

Fig. 8 Variation of $\omega_{h o}{ }^{2} / \omega_{O}{ }^{2}$ ratio to changes of $\varepsilon=[0,1]$, various $P_{2} / P_{1}, b / a=1, E_{2} / E_{1}=1$.

\section{B. The plate body is orthotropic}

It is assumed that the body of the plate is homogeneous in terms of density but inhomogeneous and orthotropic in terms of the materials and mechanical characteristics, $\left(E_{1} \neq E_{2}\right)$, i.e., the variable $\mu$ is assumed to be 0 , and the variable $\varepsilon$ is investigated based on this assumption. In this case, the angular frequency is represented by $\omega_{i o}$ and the equation will be used for its variation.

Fig. 9 depicts the curve for the variations of the powered angular frequency in the value of $E_{2} / E_{1}$ equals 1.5 (orthotropic quality). Therefore, it can be concluded that in changing the values of $E_{2} / E_{1}$ from 1 to 1.5 , the frequency ratio and not the frequency itself, will increase 1.5 percent.

$$
\frac{\omega_{i o}^{2}}{\omega_{0}^{2}}=1-\frac{\alpha_{m}^{2} P_{1}+\beta_{n}^{2} P_{2}}{D(1+0.25 \mu)}, \quad \mu=\varepsilon
$$

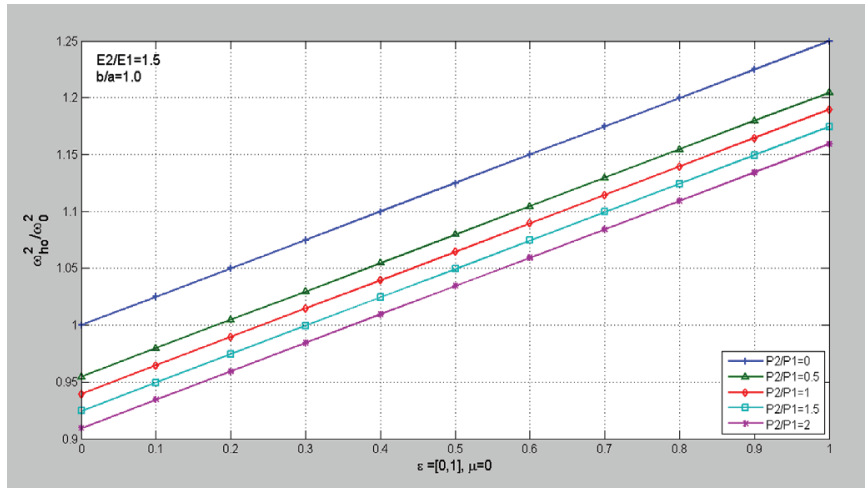

Fig. 9 Variation of $\omega_{h o}{ }^{2} / \omega_{O}{ }^{2}$ ratio to changes of $\varepsilon=[0,1]$, various $P_{2} / P_{1}, b / a=1, E_{2} / E_{1}=1.5$.

\subsection{Investigating the sensitivity of the angular frequency to the inhomogeneity variable of both the mechanical characteristics of the materials and the density, $\varepsilon, \mu$}

For the sensitivity of an angular frequency to the inhomogeneity variable of both the mechanical characteristics of materials and density, $\varepsilon, \mu$, it is assumed that the body of the plate is inhomogeneous in terms of density and in terms of materials and mechanical characteristics; it also has an orthotropic quality, i.e., the variables $\varepsilon$ and $\mu$ are investigated based on this assumption. For simplicity, it is 
assumed that $\varepsilon=\mu$. In this case, the angular frequency is represented by $\omega_{i 0}$. The first index $(i)$ indicates the inhomogeneity of the density and mechanical characteristics, and the second one represents the orthotropic quality.

The following will analyze the sensitivity of the powered angular frequency ratio to the changes in the variables $\varepsilon$ (the inhomogeneity of the mechanical characteristics of the materials) and $\mu$ (inhomogeneity of density). Equation has a nonlinear relationship with the variables $\mu$ and $\varepsilon$; therefore, the powered ratio of the angular frequency will vary in a nonlinear fashion. Fig. 10 depicts the curve for the variations of the powered angular frequency to the density inhomogeneity and inhomogeneity of the mechanical characteristics of the materials' parameters, in various ratios of $E_{2} / E_{1}$ and $P_{2} / P_{1}$. For a square plate the frequency ratio will decrease $3 \%$ to $4 \%$ by an increase in $P_{2} / P_{1}$ of $100 \%$, the moduli ratio $E_{2} / E_{1}$ is constant. The Fig. depicts this softening in a square plate $(b / a=1)$ for the various values of $P_{2} / P_{1}$.

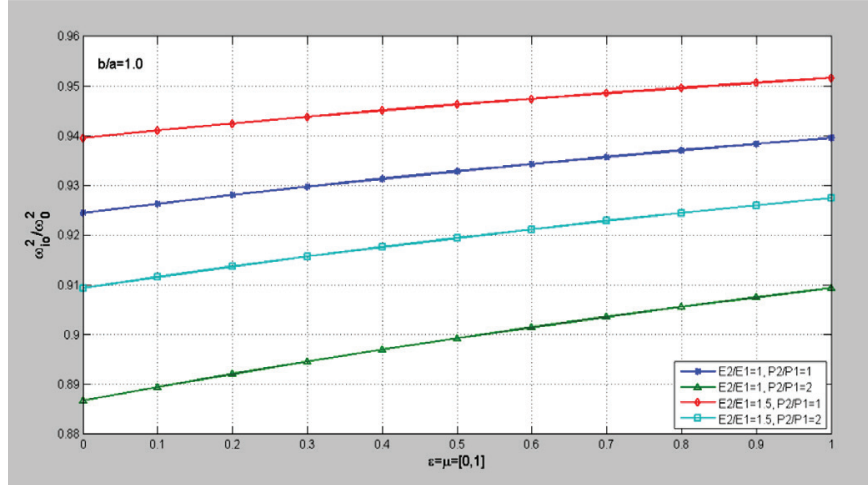

Fig. 10 Variation of $\omega_{i o}{ }^{2} / \omega_{O}{ }^{2}$ ratio to changes of $\varepsilon=\mu=[0,1]$, various $P_{2} / P_{1}$ and $E_{2} / E_{1}$.

\subsection{Investigating the sensitivity of the angular frequency to the higher vibration modes, $m=n>1$}

For the sensitivity of the angular frequency in higher vibration modes, $(m=n>1)$ to the variables $\varepsilon$ and $\mu$, an analysis of their sensitivity was conducted, and the results are presented in Fig. 11. Fig. 11 depicts the curve for the variations of the powered angular frequency to the variables $\varepsilon$ and $\mu$, in various amounts of $m=n=1$ and $m=n=2$, which decrease in the powered angular frequency with an increase in the $m=n$ values. On the other hand, the sensitivity of the powered angular frequency to changing the variables $\varepsilon$ and $\mu$ is larger in the first vibration mode and not in the second and third ones.

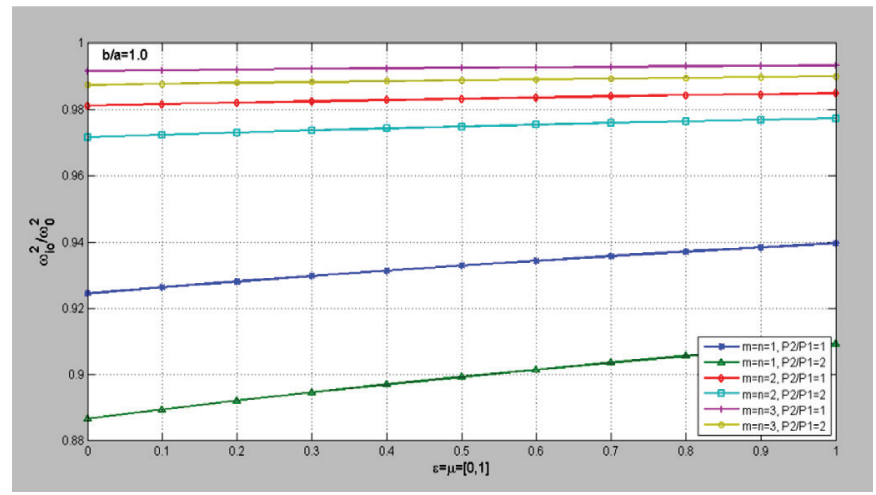

Fig. 11 Variation of $\omega_{i o}{ }^{2} / \omega_{O}{ }^{2}$ ratio to changes of $\varepsilon=\mu=[0,1]$ in the higher vibration modes.

\section{CONCLUSION}

Among the variables examined in this study, the cross-section geometry, the length and width of the plate and the loads applied to the plate as the first group (with certainty), and the variables of the mechanical properties, including density, elasticity modulus, shear modulus, the modulus of elasticity of the two main directions and the effective thickness of the plate as the second group (with uncertainties) are estimated.

Various methods for the approximate estimation of the second group of variables have been presented by various researchers and codes. One of the areas of this research is estimating the frequency errors based on the uncertainties of the second group of parameters. For example, in estimating the modulus ratio (orthotropic quality) that there is approximately $15 \%$ of uncertainty, the probability of a few percentages of error in determining the frequency ratio would not be far-fetched. Or, if in determining the inhomogeneity of the mechanical properties to be confronted with a $5 \%$ uncertainty in the desired frequency ratio output, a few percentages of error would not be far-fetched.

In this study, the effect of material uncertainties on the free vibration of inhomogeneous orthotropic concrete plates was investigated. Material properties affect the critical values of the free vibration frequencies of plates. The material uncertainty was represented by the most important parameters of concrete, i.e., concrete strength and the elastic modulus. Using the same mixing, concrete could achieve different compressive strength results in different situations. In practice, with a change in gradation and concrete compaction, the density and the compressive strength of concrete are changed.

To determine the uncertainty and sensitivity of every defined variable (inhomogeneity of density, inhomogeneity of the mechanical characteristics of materials such as $E$ and $G$, orthotropic quality, changes in plate dimensions, changes in the ratio of length to width $b / a$, and changes in applied forces in two main directions $\left.\left(P_{2} / P_{1}\right)\right)$, the sensitivity of the parameters was analyzed and the results presented.

Based on a hyper-geometric solution, the numerical values of the free vibration frequencies of inhomogeneous orthotropic concrete plates are computed and presented.

This work was run as a Ph.D. project, and this research was conducted from 8/2012 to 6/2013. The Research Project was partially sponsored by the "FARBANA Consulting Engineers". 


\section{REFERENCES}

ACI-CRC Final Report, October (2008) A Study of Static and Dynamic Modulus of Elasticity of Concrete.

Bazant, Z.P. (2000) Structural stability. International Journal of Solids and Structures, No. 37.

Berczyński, S. - Gutowski, P. - Kravtsov, Y. - Chodźko, M. (2011) Estimation of nonlinear models' parameters of machine tool supporting systems using incomplete vibration test data. Advances in Manufacturing Science and Technology, 5-18.

Department of Environment and Conservation (NSW) (2006) $\mathrm{As}$ sessing Vibration: a technical guideline. ISBN 1741378125

Elishakoff, I. (2005) Eigenvalues of Inhomogeneous Structures. CRC Press.

Ellingwood, B.- A. Tallin (1984) Structural Serviceability: Floor Vibrations. Journal of Structural Engineering, Vol. 110, No. 2, (C)ASCE.

Mali, K.D. - Singru, P.M. (2012) Analytical model to determine fundamental frequency of free vibration of perforated plate by using unit step functions to express non-homogeneity. Journal of Vibro Engineering. Vol. 14, No 3. ISSN 1392-8716

Krauberger, N. - Bratina, S. - Saje, M. - Schnabl, S. - Planinc, I. (2012) Inelastic buckling load of a locally weakened reinforced concrete column. Engineering Structures, vol. 34, 278-288.

Lal, R.- Kumar, Y.- Gupta, U. S. (2010) Transverse Vibration of Non-Homogeneous Rectangular Plates of Uniform Thickness Using Boundary Characteristic Orthogonal Polynomials. Int. Jl. of Applied Mathematics and Mechanics, Vol. 6, pp. 93-103.

L. Shahsavar, V. (2011) Forced vibration of non-uniform orthotropic inhomogeneous rectangular plates clamped in two sides. Theoretical and Applied Mechanics, AZUAC, Azerbaijan University of Architecture and Construction, Vol. 21, No. 1.
Luo, Y. - Li, A. - Kang, Z. (2012) Parametric study of bonded steelconcrete composite beams by using finite element analysis. Engineering Structures, 34, 40-51.

Svinkin, M.R. (2004) Minimizing Construction Vibration Effects, practice periodical on structural design and construction. (C)ASCE.

Mostofinejad, D. (2006) Reinforced Concrete Structures. Arkaneh danesh, Isfahan University, 1st edition.

Pavic, A. - P. Reynolds (2007) Vibration Serviceability of Long-Span Concrete Building Floors: Part 1 - Review of Background Information. University of Sheffield, Department of Civil and Structural Engineering.

Sharma, S. - Gupta, U.S. - Singhal, P. (2012) Vibration Analysis of Non-Homogeneous Orthotropic Rectangular Plates of Variable Thickness Resting on Winkler Foundation. Journal of Applied Science and Engineering, Vol. 15, No. 3, pp. 291-300.

Hosseini-Hashemi, Sh. - Rokni Damavandi Taher, H. - Akhavan, H. - Omidi, M. (2010) Free vibration of functionally graded rectangular plates using first-order shear deformation plate theory. Applied Mathematical Modelling 34, 1276-1291.

Yin, S. - Yu, T. - Liu, P. (2013) Free Vibration Analyses of FGM Thin Plates by Isogeometric Analysis Based on Classical Plate Theory and Physical Neutral Surface. Advances in Mechanical Engineering, Hindawi Publishing Corporation, Available on http://dx.doi.org/10.1155/2013/634584.

Smith, A. - Hick, S. - Devine, P. (2007) Design of Floors for Vibrations: A New Approach. SCI Publication P354, Ascot. 